\title{
A Possible Delimitation between Facts AND STATES OF AFFAIRS
}

\author{
ANA Clara POLAKOF
}

\begin{abstract}
We argue that it is possible to differentiate facts from states of affairs ontologically. We defend a Platonist ontology, based on Chateaubriand (2001 and 2005), that is hierarchized in levels and types. It contains concrete objects as the most basic entities, logical and non-logical properties, facts, states of affairs, among other entities. While some current philosophers (such as Chateaubriand 2001 and Armstrong 1997) do not distinguish facts from states of affairs, we argue that this is not how they should be treated. We consider that, even though they are both instantiated entities, they have different features. We are convinced that, in a hierarchized ontology, facts and states of affairs should occupy different levels, have different types, as well as different characteristics. In this article, we will show what is the distinction we propose, as well as we what are the advantages that our distinction possess.
\end{abstract}

Keywords: Chateaubriand; facts; states of affairs; ontology.

\section{Introduction}

There are different ways to approach the problem of whether or not facts should be differentiated from states of affairs: you may not distinguish them (as Armstrong 1997 and Chateaubriand 2001 do), you may take the possible worlds alternative and say that facts are obtained states of affairs (see Reicher 2009, p.27), or you may even say that they are not necessary entities (Simons 2009). ${ }^{1}$ If we narrow it down to the two alternatives that consider that there are states of affairs, we may see that they share some problems. They are both subject to Bradley's regress and, even within the possible worlds alternative, no agreement has been reached with respect to how these entities are formed. ${ }^{2}$ Some argue that states of affairs are constituted by an internal relation, others that they are the relation (or connector), and others argue that the relation must be external (see Valicella 2000, p.238). So, the traditional theory of states of affairs that postulates that states of affairs are extra-linguistic entities that "serve as the descripta of sentences (closed formulae)" (Taylor 1976, p.263) has not yet been able to untangle what states of affairs are, and the proposals provided by Armstrong (1997) and Chateaubriand (2001) are not entirely problem-free, which is why an alternative should be provided.

We want to argue that it is possible to differentiate facts from states of affairs as instantiated entities. ${ }^{3}$ We develop our proposal within Chateaubriand's ontology

Principia 20(3): 361-376 (2016).

Published by NEL — Epistemology and Logic Research Group, Federal University of Santa Catarina (UFSC), Brazil. 
which is a hierarchized ontology that contains as the most basic entities level 0 concrete objects, and starting from level 1 it contains abstract entities such as properties and states of affairs. We think that within this ontology, in which facts are not distinguished from states of affairs, it is possible to differentiate them. This differentiation may be questioned, as one of the reviewers noted. However, we are convinced that this difference gives a correct account of a logical ontology as the one Chateaubriand proposes. The difference we propose, as we will show, is based on the levels they occupy, the ontological characteristics they have, and the types they have. Even though we defend an ontology which only has entities that are there (as Chateaubriand does), this does not mean that our proposal may not be extended to a possible worlds framework for the cases of obtained states of affairs (that is, facts). This means taking into account many issues that are not considered in this paper. We do not, for instance, give any account of modality. Modality has been crucial in the definition of states of affairs, as one of the reviewers correctly pointed out. However, we are proposing a new definition of states of affairs that is independent of modality, and independent of the way we usually speak about states of affairs. ${ }^{4}$

In this article, we propose two different approaches to the delimitation of facts and states of affairs. The first tries to differentiate facts from states of affairs according to the levels they occupy in the hierarchy. We show that this approach has several problems which force us to abandon it. This is due to the fact that the level the entities occupy in the hierarchy is not sufficient to differentiate them. We, then, suggest a second approach. It takes into account some characteristics that were not considered in the first, which make it a better alternative to differentiate facts from states of affairs. Given that levels do not differentiate the entities, types need to be introduced. We consider that our second approach effectively differentiates facts from states of affairs, and should be taken into account by philosophers who wish to deal with logical entities.

\section{A first approach to a possible delimitation between facts and states of affairs}

Chateaubriand proposes a complex realist ontology that has objects, which are concrete entities, and properties and states of affairs, which are abstract entities. These entities are organized according to an ontological hierarchy in which objects are at the lowest level of the hierarchy and properties and states of affairs are at all levels except 0 , this is to say that they may occupy all levels from level 1 and up. ${ }^{5}$ Objects are the only concrete entities, and the most basic entities of the ontology. ${ }^{6}$ Properties are abstract entities that pertain to levels 1 and up, and they may be logical or nonlogical. Some properties may appear in all abstract levels (for instance, the logical 
property Existence appears from level 1 and up), others may appear only in one abstract level (for instance, being blue appears on level 1 alone), and others may appear in several levels but not all (for instance, the logical property Universal Subordination appears from level 2 and up). Chateaubriand's states of affairs are defined as combinations or instantiations of a property in an object or objects and/or in a property or properties. For instance, the instantiation of being blue in my bike results on a level 1 state of affairs, the instantiation of Universal Subordination in the properties being human and being mortal results on a level 2 states of affairs, the instantiation of Existence3 in Universal Subordination results on a level 3 states of affairs, and so on.

Properties are essential to Chateaubriand's proposal, because states of affairs result from the instantiation of properties in other entities (they may be objects and/or properties). Chateaubriand (2013, p.102) defines a property as an abstract entity which is designated by a predicate and may or may not be applied to another entity. These properties allow us to identify any kind of entities (they may be concrete, like a cat, or they may be abstract, like transitivity); and they contain identity conditions (Chateaubriand, 2013, p.102). ${ }^{7}$ According to Chateaubriand we may have logical and non-logical properties. Non-logical properties are those that do not depend on logic. We have non-logical properties (such as being brown or being a philosopher) at level 1, other non-logical properties (such being a level 1 state of affairs) at level 2 , and at higher levels (such as being a level 2 state of affairs, being a level 3 state of affairs, and so on). Logical properties are universal, significant in all the hierarchy, and like Diversity they may appear on different levels of the hierarchy. ${ }^{8}$ It is the existence of logical properties that leads us to a hierarchized ontology which cannot have an upper bound. For, as Whitehead and Russell (1910) showed, if it weren't hierarchized and if it had an upper bound, logical paradoxes would arise. So, logical properties may appear at level 1 (Existence1) and in higher levels (Existence2 at level 2, Existence3 at level 3, and so on). ${ }^{9}$ This does not mean that they are all one and the same property, if they were they would not pertain to different levels and have different types. They instantiate in different entities and give rise to different states of affairs which also appear in levels equal or higher than 1.

Our proposal takes Chateaubriand's ontology as a starting point, and agrees with it in some essential characteristics: both facts and states of affairs are abstract entities, and they both result from the instantiation of a property in another entity. However, we propose that, while they do share those characteristics, there is an essential difference between them: facts result from the instantiation of a property in a concrete object or objects while states of affairs result from the instantiation of a property in another property or properties. This difference, as we will try to show, will have a direct repercussion in their logical and ontological characteristics.

Our first approach proposes that it is possible to differentiate the entities accord- 
ing to the levels they occupy in the hierarchy. That is, since facts involve the instantiation of a property in an object or objects, they will occupy a lower level than states of affairs. We could, then, state that facts are level 1 entities in which a level 1 property is instantiated in level 0 objects; and states of affairs are level 2 and up entities in which a property of level higher or equal to 2 is instantiated in a property or properties that are at least one level lower than it (in the case of a level 2 property, it will be instantiated in a level 1 property). States of affairs have to be level 2 or higher because the lowest level in which properties may combine with properties is level 2, and facts have to be level 1 because they are the instantiation of a property in an object or objects. Let's assume, for instance, that Existence is a logical property which appears at level 1 and is instantiated in a level 0 object; it then appears in level 2 (because it is a cumulative hierarchy) and is instantiated in a level 1 property; it appears in level 3 and is instantiated in a level 2 property; and so on. The instantiation of Existence1 in a level 0 object like my dog results in the fact $\langle$ Existence1, my dog , while the instantiation of Existence 2 in a level 1 property like Diversity 1 results in the state of affairs $\langle$ Existence2, Diversity 1$\rangle .{ }^{10}$ If this first approach were to be correct, it would allow us to differentiate between facts and states of affairs according to the level they occupy in our hierarchy. It would also separate those instantiations of properties in objects from those instantiations of properties in properties. This would constitute, for us, an important advantage with respect to treating them as the same kind of entity, because higher-level entities would be treated as different entities than lower-level ones. This would, for instance, allow us to differentiate entities that could be accepted by a nominalist (such as level 1 facts) ${ }^{11}$ from entities that would never be accepted by a nominalist because they are higher-level entities (such as state of affairs). ${ }^{12}$

Within this approach, and the one we will consider next, time has to be taken into account. It may be seen as a necessary condition to distinguish properties from objects: level 0 objects may be characterized as temporal entities, while properties may be characterized as atemporal entities. ${ }^{13}$ Objects are temporal because there could be times at which they did not exist, while properties are atemporal because they exist always. That is, within our approach properties are Platonic universals that are always there and do not need to be instantiated to exist. ${ }^{14}$ Of course, someone could argue that being mortal is a temporal property determined by mortality, but this would be mistaking the abstract property of being mortal with the fact that, for instance, an object of level 0 is mortal. We may, then, argue that facts - such as $\langle$ being mortal, my $\operatorname{dog}\rangle$ - are what are temporal, and they are made temporal because of the object. ${ }^{15}$ The property maintains its atemporality. On the other hand, states of affairs are atemporal because they do not depend on temporal entities, for they are the instantiation of an atemporal entity (a property) in atemporal entity/ies (other property/ies). This is why states of affairs like $\langle$ Existence3, Diversity 2$\rangle$, and 〈Universal 
Subordination, being human, being mortal $\rangle$ appear to be more general than facts: they are all states of affairs that do not involve objects and are, thus, more general. We are convinced that temporality should be taken into account if we want to differentiate what is dependent upon an object and what is dependent upon a property, and that it is possible to assert that facts are temporal while states of affairs are atemporal.

The last advantage we want to consider in this section has to do with the recognition/proposal that there are logical and non-logical properties. On the one hand, if we were to maintain that facts are the same entities as states of affairs, an heterogeneous treatment of those entities would arise regarding the combination of logical and non-logical properties. This is due to the fact that, since we have logical and non-logical properties, we have to accept that we may have logical and non-logical facts or states of affairs (cf. Chateaubriand 2013). They will be logical if the combination is between logical properties, and they will be non-logical if there is at least one non-logical entity involved in the combination. We would have to accept, then, that all level 1 states of affairs would be non-logical because they combine with a level 0 object, and that only from level 2 and up we would have (in addition to non-logical states of affairs as (Universal Subordination, being human, being mortal $\rangle$ ) logical states of affairs (such as $\langle$ Existence2, Diversity 1$\rangle$ ). On the other hand, separating the entities that are always non-logical (as level 0 objects) from the entities that may be logical or non-logical (as properties) may be considered an advantage. The difference we propose allows us to make a more homogeneous treatment of facts: facts will always be non-logical. This assimilates facts to level 0 objects which are also non-logical. However, states of affairs continue to be heterogeneous: they may be logical or nonlogical. This assimilates states of affairs to properties which may also be logical or non-logical. Our proposal lets us show that, not only are facts similar to objects from a temporality point of view, they are similar to objects in that they are non-logical. It also allows us to show that, not only are states of affairs similar to properties in that they are atemporal, they are also similar to properties in that they may be logical or non-logical.

To summarize, this first approach allows us to differentiate facts from states of affairs which, we consider, is an advantage to treating all these entities as equal (facts as being the same as states of affairs). First, we defend that it is possible to differentiate facts from states of affairs according to the level they occupy in the ontological hierarchy. Facts are level 1 entities, while states of affairs are level 2 and up entities. Second, we defend that it is possible to differentiate facts from states of affairs with regards to their temporality. Facts are temporal entities because they are the instantiation of a property in a temporal object or objects, while states of affairs are atemporal because they are the instantiation of an atemporal property in an atemporal property or properties. ${ }^{16}$ Third and last, we defend that the differentiation between facts and states of affairs allows us to make a more homogeneous treatment of facts. Facts are 
always non-logical entities because they involve level 0 objects, while states of affairs may be logical (if they combine logical properties) or non-logical (if they involve at least one non-logical property) because they may involve logical or non-logical properties. There are, however, some problems with our first approach that we consider in the next section.

\section{Problems with the first approach}

The first approach proposes that facts may be differentiated from states of affairs according to the levels they occupy. However, some problems arise if we maintain facts as level 1 entities. These problems are related to the definitions initially given for the entities, and to the time condition which stated that facts were temporal while states of affairs were atemporal. The first problem is that, even though we defined facts as the result of properties being instantiated in objects, and states of affairs as being those instantiated in properties, properties of level 2 and higher should be able to be instantiated in facts or states of affairs. Both entities could perfectly be arguments of a higher level property, because we work within a cumulative hierarchy. Thus, we should have properties of level 2 or higher that could be instantiated in level 1 facts. We could have a level 2 logical property like Existence 2 instantiated in a fact like $\langle$ being mortal, my $d o g\rangle$. We would, then, have to say that this is a state of affairs because it is a level 2 entity, and we should redefine states of affairs as being the result of the instantiation of a property in properties and/or facts or states of affairs (if it is in a level equal or higher than 3). This would, apparently, solve the first problem. However, a second problem arises with this definition. It seems that, if the example we gave were a state of affairs, we would have a temporal state of affairs, since the fact in which the property is instantiated is temporal. If we were to maintain this, we would lose one of the important distinctions between facts and states of affairs: facts are temporal and states of affairs are not, which is not a price we want to pay. We want to hold that facts are temporal and states of affairs are atemporal. We think that, if we accept that states of affairs could also be temporal, the distinction between facts and states of affairs would really be merely terminological: we call the complex level 1 entities facts, the others states of affairs, and there is no ontological difference between them. This is not what we want to defend.

So, the first problem could be solved by adding an extra part to the definition of states of affairs, but the second problem seems to be more difficult to resolve, and our proposal needs to be revised. Facts have to be temporal entities, and states of affairs atemporal entities. The difference, then, may no longer be a clear cut difference of the entities that depends only on the level they occupy, it has to be found somewhere else. It is not in the abstract/concrete distinction, for as we mentioned, we agree with

Principia 20(3): 361-376 (2016). 
Chateaubriand in that they are both abstract entities; nor is it in the obtained or not obtained distinction of the possible world framework because, for us, they are both obtained. To solve this problem we have to accept that we have facts in all levels equal or higher than 1 , and that we have states of affairs in levels equal or higher than 2 . We can still argue that only facts are level 1 entities, temporal and always non-logical; while states of affairs continue to be level 2 and up entities, atemporal, and logical or non-logical; but they need to be redefined as:

- Facts are level 1 and up entities which involve the instantiation of a property in an object or objects. We will, then, have: ${ }^{17}$

- level 1 facts such as $\langle$ being a philosopher, Chateaubriand $\rangle$; 〈being mortal, my dog $\rangle$, 〈Existence1, my dog $\rangle$, 〈Diversity1, Chateaubriand, my dog $\rangle$, etc.

- level 2 facts such as $\langle$ Existence2, 〈being a philosopher, Chateaubriand $\rangle\rangle$; $\langle$ Diversity2, 〈being a philosopher, Chateaubriand $\rangle,\langle$ being mortal, my dog $\rangle$, $\langle m y \operatorname{dog}\rangle\rangle$, etc.

- level 3 facts such as $\langle$ Existence3, 〈Existence2, 〈being a philosopher, Chateaubriand $\rangle\rangle\rangle$; $\langle$ Diversity3, $\langle$ Diversity2, $\langle$ being a philosopher, Chateaubriand $\rangle$, 〈being mortal, my dog $\rangle,\langle$ Diversity 1 , Chateaubriand, my dog $\rangle\rangle,\langle$ Existence2, 〈being a philosopher, Chateaubriand $\rangle\rangle$, 〈being mortal $\rangle\rangle$, etc.

$\circ$ and so on.

- States of affairs are level 2 and up entities which result from the instantiation of a property in property/ies (not instantiated in object/s) and/or in state/s of affairs (for level 3 states of affairs and up). ${ }^{18}$ We will have, then:

- level 2 states of affairs such as $\langle$ Existence2, Diversity 1$\rangle ;\langle$ Universal Subordination, being human, being mortal $\rangle$; etc.

- level 3 states of affairs such as $\langle$ Existence3, Diversity2 $\rangle ;\langle$ Diversity3, Diversity2, Existence2, 〈Universal Subordination, being human, being mortal $\rangle,\langle$ Existence2, Diversity 1$\rangle\rangle$, etc. ${ }^{19}$

$\circ$ and so on.

In this second approach, we may not differentiate the entities according to the levels, but it is still possible to maintain that facts may be level 1 entities, while states of affairs may only be level 2 and up. Even though we lost the clear differentiation regarding levels, we can maintain the differentiation regarding temporality: facts are temporal entities, while states of affairs are atemporal entities. And nothing is lost with respect to the logicality of the entities: facts are always non-logical because they always involve a non-logical entity, while states of affairs may be logical or non-logical depending on the properties that are involved in the state of affairs. 
It could now be stated that a nominalist would no longer accept our facts, since they no longer pertain to level 1 alone, and that one of our arguments for maintaining that the difference is important is lost. ${ }^{20}$ This would be a valid criticism, for it is sure that an ostrich nominalist would not accept higher level facts, and since we were forced to accept those facts as well as level 1 facts, our distinction is not relevant at all. Perhaps, here, the notion of ground (as used in Fine 2012) could be of some assistance: facts are always grounded (in some way or another) in objects, while states of affairs are never grounded in objects, they are grounded in properties that are not instantiated in objects. A nominalist could accept that, since facts are grounded in objects, they exist. He could say that higher level facts are not necessary and are, thus, reducible to the facts that have objects as immediate grounds (that is level 1 facts). A nominalist could then say that states of affairs are not there at all. They do not need to be reduced, because - since their grounds do not exist - they do not exist. And so, our distinction is maintained: facts involve the instantiation of a property in an object, while states of affairs involve properties that are not instantiated in objects.

These entities are important to an ontology like the one proposed by Chateaubriand: the distinction between them allows us to differentiate temporal entities from atemporal ones, and it allows us to separate what is logical from what is non-logical which, we think, is very important. If we accept that logical entities exist, we should accept that it is important to differentiate them from what is not logical; and our distinction allows us to do just that. Within this second approach a simple distinction according to levels is not possible, and even though we have argued that they are ontologically different entities, no adequate account in the hierarchy has been provided. It is not sufficient to say that facts are level 1 and up entities, and states of affairs are level 2 and up entities. We have to provide a further distinction, and this is why types become relevant: the entities may still be differentiated according to their types, as we will show in the next section.

\section{The type of facts and of states of affairs}

As we mentioned, given that the level is not a sufficient condition to differentiate facts from states of affairs, we need to take something else into account: their types. For us to do this, we need to briefly summarize Chateaubriand's hierarchy. ${ }^{21}$ Chateaubriand is inspired by Frege and Russell in proposing his hierarchy. Frege's hierarchy involved saturated level 0 objects, and unsaturated functions of first and second order. Frege's hierarchy, as is well known, involved paradoxes. Russell's and Whitehead theory of types arose to account for those paradoxes. The basic idea was that reality had to be structured in a hierarchy, which restricted the arguments that a propositional func-

Principia 20(3): 361-376 (2016). 
tion of level n could take, to avoid contradictions. This idea can be seen clearly in the introduction of Principia Mathematica and in Russell (1908). In both works, contradictions that may be described as due to self-reference or reflexiveness (we have the cases of the true statement about lying; the classes of members that are not members of themselves; Richard's Paradox; among others) are introduced and the theory of types postulated as the theory that effectively solves these contradictions. ${ }^{22}$

Chateaubriand considers, as Frege and Russell did, that objects or individuals are at the lowest level 0 and they are defined as everything that is not a property or a state of affairs (Chateaubriand 2001, p.301). At level 1 we will have logical properties such as Diversity, Identity, Existence, Non-Existence, and the possible combinations between them construed by logical operations; non-logical properties as being a dentist, being human, etc.; and states of affairs that result from the combination of those properties with level 0 object/s. Starting from level 2 we have logical properties (which are infinitely many, according to Chateaubriand (2013, p.103)), non-logical and logical states of affairs. The hierarchy, as mentioned, is flexible and cumulative which means that properties may have a variable arity (they may be instantiated in a different number of entities), appear in different levels, and take different entities as their arguments. Only properties have a variable arity, objects and states of affairs do not. To reflect this differences typologically, Chateaubriand proposes a type theory that types all the entities that exist in his ontology. The type of the entities is defined by their level and their arity (if we are dealing with properties or instantiated properties which have arity); and is used to differentiate logically the ontological entities. The type will differ depending on the level, on the entities they may take as arguments, and on the entities in which they are instantiated. As the complexity of the entities grows, the complexity of the type also grows. To know their types, it is possible to start from the lowest level and reach to a complex property of level $\lambda$ and arity $\kappa$ which, if instantiated, will give a state of affairs of the same level. The simplest type is the one that objects have, 0 . Then, the type of the properties comes. He proposes a general form for properties in which $\lambda$ is the level of the property, $\kappa$ is the arity of the property, and $\tau$ are the types of the entities that the property takes as arguments $\left\langle\langle\lambda, \kappa\rangle, \tau_{0}, \tau_{1}, \ldots\right\rangle$. In the case of the state of affairs, the type will be even more complex because it needs to include the entities in which the property is instantiated: $\left\langle\left\langle\langle\lambda, \kappa\rangle, \tau_{0}, \tau_{1}, \ldots\right\rangle, \tau_{0}, \tau_{1}, \ldots\right\rangle$. The types of the entities, in Chateaubriand's hierarchy, are:

- objects pertaining to level 0 are of type 0

- properties pertaining to level 1 will have different types depending on the number of objects they apply to: $\left\langle\left\langle 1, \kappa_{1}\right\rangle, 0,0, \ldots\right\rangle$

- states of affairs of level 1 will have the type of the property being instantiated as well as the objects in which the property is instantiated: $\left\langle\left\langle\left\langle 1, \kappa_{1}\right\rangle\right.\right.$, 
$0,0, \ldots\rangle, 0,0, \ldots\rangle$

- properties pertaining to level 2 will have different types depending on the number of properties they apply to: $\left\langle\left\langle 2, \kappa_{2}\right\rangle,\left\langle\left\langle 1, \kappa_{1}\right\rangle, 0,0, \ldots\right\rangle, \ldots\right\rangle$

- states of affairs of level 2 will have the property being instantiated as well as the properties in which the property is instantiated: $\left\langle\left\langle\left\langle 2, \kappa_{2}\right\rangle,\left\langle\left\langle 1, \kappa_{1}\right\rangle\right.\right.\right.$, $\left.\left.0,0, \ldots\rangle, \ldots\rangle\rangle,\left\langle\left\langle 1, \kappa_{1}\right\rangle, 0,0, \ldots\right\rangle, \ldots\right\rangle, \ldots\right\rangle^{23}$

- And so on, up to infinity, because the hierarchy has no upper bound.

What we propose is a little bit different, but only because we propose a distinction between facts and states of affairs. That is, the difference is only in the types that facts and states of affairs have:

- objects and properties maintain their types ${ }^{24}$

- facts will have varying types according to their levels, but they will involve at least the type of an object or the type of a level 1 fact $\langle\langle\langle 1, \kappa\rangle$, $0,0, \ldots\rangle, 0,0, \ldots\rangle$. A general type for facts of level higher than 1 could be: $\left\langle\left\langle\langle\lambda, \kappa\rangle, T_{0}, T_{1}, \ldots\right\rangle, T_{0}, T_{1}, \ldots\right\rangle$ where at least one $T$ involves that of an object or that of a level 1 fact.

- states of affairs will also have varying types, but none of them will have in their types the type of a level 1 fact nor that of an object, because if they did they would be facts. A general type for states of affairs could be: $\left\langle\left\langle\langle\lambda, \kappa\rangle, \tau_{0}, \tau_{1}, \ldots\right\rangle, \tau_{0}, \tau_{1}, \ldots\right\rangle$ where $\tau$ may never involve that of an object nor that of a fact. ${ }^{25}$

We may now give some examples that make the difference clearer. Let's start with facts. A level 1 fact such as $\langle$ being mortal, my dog $\rangle$ would have the type $\langle\langle\langle 1,1\rangle, 0\rangle, 0\rangle$ because it is instantiated in one object that has 0 as its type. A level 2 fact such as $\langle$ Diversity2, 〈being a philosopher, Chateaubriand $\rangle$, $\langle$ being mortal, my dog $\rangle\rangle$ would have the type $\langle\langle\langle 2,2\rangle,\langle\langle\langle 1,1\rangle, 0\rangle, 0\rangle,\langle\langle\langle 1,1\rangle, 0\rangle, 0\rangle\rangle,\langle\langle\langle 1,1\rangle, 0\rangle, 0\rangle,\langle\langle\langle 1,1\rangle, 0\rangle, 0\rangle\rangle$; where the first 2 is the level of the property, the second is the arity of the property. Then, we have the types of two level 1 facts which give us the type of the property, and the types of those facts repeated which gives us the information that the property was instantiated in those facts. We are, then, in front of a fact and not in front of a property. A level 3 fact such as $\langle$ Existence3, 〈Existence2, $\langle$ being a philosopher, Chateaubriand $\rangle\rangle\rangle$ would have the type $\langle\langle\langle 3,1\rangle,\langle\langle\langle 2,1\rangle,\langle\langle\langle 1,1\rangle$, $0\rangle, 0\rangle\rangle,\langle\langle\langle 1,1\rangle, 0\rangle, 0\rangle\rangle\rangle,\langle\langle\langle 2,1\rangle,\langle\langle\langle 1,1\rangle, 0\rangle, 0\rangle\rangle,\langle\langle\langle 1,1\rangle, 0\rangle, 0\rangle\rangle\rangle$; and so on. Evidently, the types of the facts become more complex when we start to go some levels up. However, we may now see that all these have in their types a level 1 fact embedded in the complex type, and, thus, respect the definition given for facts.

Let's continue with states of affairs, which start from level 2 and go up. The type of a level 2 state of affairs such as $\langle$ Diversity2, being a philosopher, being a dentist $\rangle$, would 
be: $\langle\langle\langle 2,2\rangle,\langle\langle 1,1\rangle, 0\rangle,\langle\langle 1,1\rangle, 0\rangle\rangle,\langle\langle 1,1\rangle, 0\rangle,\langle\langle 1,1\rangle, 0\rangle\rangle$. You may notice that, since it does not involve the instantiation of the properties in objects, the type is simpler than

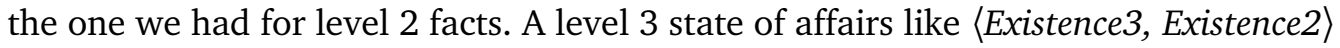
would have the type $\langle\langle\langle 3,1\rangle,\langle\langle 2,1\rangle,\langle\langle 1, \kappa\rangle, 0,0 \ldots\rangle\rangle\rangle,\langle\langle 2,1\rangle,\langle\langle 1, \kappa\rangle, 0,0 \ldots\rangle\rangle\rangle$, which corresponds to the property Existence 3 being instantiated in the uninstantiated property Existence2. $\kappa$ is there because Existence2 may take as an argument any level 1 property, which is a result of the cumulative nature of the hierarchy. ${ }^{26}$ The examples get even more complex as we go some levels up. However, it is possible to see that the type of a level 1 fact cannot be found in either of them, which allows us to differentiate them from facts and, thus, the definition we gave for states of affairs is also respected.

We know that in this second approach, the typological part became more complex for facts at least, but nevertheless the differences remain and are sharp in it (as may be seen from the examples). Even though the types become more and more complex, they allow us to differentiate ontological categories very clearly. Perhaps, it is not the easiest alternative, but it allows us to classify the entities and that is important to us. ${ }^{27}$ Obviously we do not convince a nominalist with this, but we may convince someone that works within the possible world framework: even within those obtained states of affairs (or facts) we have to differentiate that that depends upon an object from that that depends upon a property, that which is temporal from that which is atemporal.

\section{Final remarks}

We hope to have shown that it is possible to differentiate facts from states of affairs, and that this differentiation is worthwhile. We hope to have shown that, even though our first approach was not satisfactory, it is possible to provide a satisfactory approach to the difference. Facts may appear at level 1, while starting from level 2 and up we may have facts and states of affairs that may be differentiated through their ontological characteristics and their types. We are convinced that our distinction involves some natural intuitions about the characteristics of facts and states of affairs: since the former involve properties instantiated in objects they are temporal, and since the latter involve properties that are not instantiated in objects they are atemporal. We also argued that this difference allows us to hold that facts are always non-logical, while states of affairs may be logical (if they combine logical properties) or non-logical (if they involve at least one non-logical property).

Even though we had to abandon the idea that the entities could be differentiated only with respect to the level they occupied, we argued that our distinction was still sound. We may still defend that facts are temporal, non-logical, that they appear in the hierarchy at level 1 ; and that states of affairs are atemporal, logical or non-logical, 
and that they appear in the hierarchy at level 2 . We do not think that in abandoning the clear cut difference of levels between the entities we lost explanatory power. On the contrary, we gained explanatory power because we are no longer attached to levels. We may distinguish the entities solely because of their ontological and logical characteristics: facts are temporal, states of affairs atemporal; facts involve the instantiation of a property in an object or objects, states of affairs involve properties that were not instantiated in objects; facts are always non-logical, states of affairs may be logical or non-logical. All of these differences may be reflected in the hierarchy of types, in which we have different types for the different entities. Even when we go levels up, the types are still different and there is no way to confuse them, because the types of facts involve objects, while the types of states of affairs do not. We are convinced that enough evidence has been provided to defend the difference between facts and states of affairs, and hope to have shown that this difference is important for a realist ontology as the one we propose.

In conclusion, we want to defend that in an ontology like the one proposed by Chateaubriand (and even within the possible worlds framework ${ }^{28}$ ) it is possible to differentiate facts from states of affairs. We also want to defend that this differentiation is advantageous with respect to treating them as the same kind of entities because it allows us to take into account some natural intuitions with regards to the entities concerned in the instantiation of the given property. And, we defend that having facts in Chateaubriand's ontology is a nice addition which allows us to further differentiate in his logical ontology what is more dependent upon logic from what is more dependent upon the concrete. We also hope to have shown that, even though we have a determinate framework, our distinction goes beyond it. A nominalist would most certainly not accept it. However, with a clear definition of the entities, he could explain why they are not there. A realist should accept the difference, or at least explain why it is not relevant. We, of course, maintain that it is relevant, and that it is a solid explanation of what is really there.

\section{Acknowledgments}

This work was possible thanks to CNPq.

\section{References}

Armstrong, D. M. 1978. Nominalism and Realism. Universals and Scientific Realism. Volume 1. Cambridge/London/New York/Melborune: Cambridge University Press.

. 1997. A World of States of Affairs. Great Britain: Cambridge University Press.
2009. Questions about States of Affairs. In: M. Reicher (ed.) 2009a. States of Affairs. Germany: Ontos Verlag, pp.39-50. 
Chateaubriand, O. 2001. Logical Forms, Part 1. Campinas: Coleção CLE.

- 2005. Logical Forms, Part II. Campinas: Coleção CLE.

. 2012. The Ontology of Mathematical Practice. Notae Philosophicae Scientiae Formalis 1(1): 80-88.

- 2013. Logical Truth and Logical Facts. In: Mircea Dimitru; Gabriel Sandu (eds.) Truth. Bucharest: The Bucharest University Press, pp.101-111.

Fine, K. 2012. Guide to Ground. In: F. Correia; B. Schneider. Metaphysical Grounding. Cambridge: Cambridge University Press, pp.37-80.

Chisholm, R. 1976. Person and object: a metaphysical study. La Salle/Illinois: Open Court Publishing Company.

Frege, G. 1964. The Basic Laws of Arithmetic. Translated and edited by Montgomery Furth. Berkeley/ Los Angeles/ London: University of California Press.

Reicher, M. 2009. Introduction. In: M. Reicher (ed.) 2009a. States of Affairs. Germany: Ontos Verlag, pp.7-38.

Rosado Haddock, G. 2008. Chateaubriand on Logical Truth and Second-Order Logic: Some Issues of Logical Forms II. Manuscrito 31(1): 163-178.

Russell, B. 1908. Mathematical Logic as Based on the Theory of Types. American Journal of Mathematics 30(3): 222-262.

Sautter, F. 2010. Chateaubriand's Realist Conception of Logic. Axiomathes 20: 357-364.

Simons, P. 2009. Why There Are No States of Affairs. In: M. Reicher (ed.) 2009a. States of Affairs. Germany: Ontos Verlag, pp.111-128.

Taylor, B. 1976. States of affairs. In: Evans; McDowell. Truth and meaning: essays in semantics. Oxford: Oxford University Press, pp.265-284.

Whitehead, A. N.; Russell, B. 1910. Principia Mathematica. Cambridge: Cambridge University Press.

Valicella, W. 2000. Three Conceptions of States of Affairs. Noûs 34(2): 237-259.

Zalta, E. 1993. Twenty-five Basic Theorems in Situation and World Theory. Journal of Philosophical Logic 22: 385-428.

\author{
Ana Clara Polakof \\ PUC-Rio \\ anaclarapo@gmail.com
}

\title{
Notes
}

${ }^{1}$ We are staying within a realist perspective. Even though Husserl's notion of states of affairs could be taken into account - and similarities have been noted with regards to Chateaubriand's notion of states of affairs (see Rosado Haddock 2008, p.176), since our work is not phenomenological, this alternative is not considered.

${ }^{2}$ Reicher (2009, pp.17-8) explains Bradley's regress and its connection to the notion of states of affairs.

${ }^{3}$ We use entities as a general term which includes objects, events, properties, facts and states of affairs. It is similar, as one of the reviewers noted, to the use of things. However, things is

Principia 20(3): 361-376 (2016). 
broader than entities. This broadness may be seen, for instance, in Chisholm's definition of it: "... I will use 'thing' in a very broad sense. Whatever there is may be said, in this sense, to be a thing: hence properties and relations are themselves things, and so are physical objects, persons and shadows" (Chisholm 1976, p.21). Since our ontology is more restricted, we prefer the use of entities.

${ }^{4}$ As that reviewer correctly points out, this leads us to a modally-free project.

${ }^{5}$ Chateaubriand's ontological hierarchy is inspired by Frege's, Russell's and Whitehead's (Frege 1964, Russell 1908, Whitehead and Russell 1910). However, it differs from them in that it is flexible and cumulative. It is flexible because properties may have a variable arity, and it is cumulative because the logical properties may appear in different levels and combine with different kinds of entities (see Sautter 2010). This hierarchy will be further explained in the section "The Type Of Facts And Of States Of Affairs".

${ }^{6}$ There are no abstract or fictional objects in the ontology. That is, there are no unicorns, no Santa Claus in the ontology. Mathematical numbers, for instance, are considered properties and not objects: "My idea (...) was essentially this: characterize numbers in terms of properties and say this is what numbers really are" (Chateaubriand 2012, p.84). There are, thus, abstract properties and abstract states of affairs.

${ }^{7}$ Even though this is not an unproblematic definition, it is the one we will use in this article.

${ }^{8}$ Diversity may not be the best example property to readers who are not familiar with Chateaubriand's proposal, as one of the reviews noted. However, it is one of the best ones to exemplify the flexibility and cumulativity of his hierarchy. Diversity is the logical property that would be equivalent to being non-identical or different.

${ }^{9}$ Note that this Existence is not the existential quantifier. It is the property of existence. It implies that the entity which exists occurs. In the case of concrete entities, it may be understood "as the property of having a location in space time" (Zalta 1993, p.404).

10 The notation we are using is pretty simple: in the first place we have the property that is being instantiated, and then we have the entities in which the property is instantiated. In these cases, when we have a fact we have a property instantiated in an object or objects (as in $\langle$ Existence1, my $\operatorname{dog}\rangle$ ), and when we have a state of affairs we have a property that is instantiated in a property or properties (as in $\langle$ Existence2, Diversity1 $\rangle$ ).

${ }^{11}$ A Quinean nominalist would most probably not accept facts.

12 We are not taking a nominalist standpoint. What we are saying is that it is worthwhile to differentiate entities that could be accepted by someone who reduces his ontology from entities that would not be accepted by the same person. Perhaps, the distinction does not seem important up to this point to a realist, but it allows us to defend the difference between facts and states of affairs.

${ }^{13}$ We assume that there are no temporal properties, even if Chateaubriand himself accepts that there may be temporal properties (Chateaubriand 2001, p.424).

${ }^{14}$ Armstrong, on the other hand, takes an anti-Platonic perspective with regards to properties for he considers that "there is no separation of particulars and universals" (Armstrong 1978, p.113). That is, properties are not independent of being instantiated. We could have another approach which took some properties as Platonic and others as Aristotelian. However, we will maintain a Platonic approach to properties.

${ }^{15}$ This is not a novel idea. Chateaubriand (2001, p.312) argues that the set \{Frege, Russell\} - state of affairs in his terminology — "is just as temporal as Frege and Russell are, in the

Principia 20(3): 361-376 (2016). 
sense that the set did not exist before both of them did, and if they don't exist now neither does the set". He, then, acknowledges that we relate the temporal features of the set or state of affairs to the temporal features that the objects have.

${ }^{16}$ If we were to maintain this first approach, we would have to defend - in reply to one of the reviewers - that both of these conditions are necessary (taken separately), but only their conjunction is sufficient for the existence of the distinction.

${ }^{17}$ Higher levels facts may involve object/s, lower level fact/s, lower level property/ies, and/or lower level state/s of affairs. Whenever an object is involved we will have a fact, even if we combine it with a state of affairs or a property, it will continue to be a fact.

${ }^{18}$ The properties are not instantiated in objects, because if they were we would be in front of a fact. Let's consider one of a reviewers example with a slight modification. I have a dog, and the dog is yellow. That the property being yellow is instantiated in my dog is a fact. The state of affairs that results from the instantiation of Existence 2 in being yellow, for instance, involves the uninstantiated Platonic universal being yellow, and not the instantiated one. States of affairs are independent of there being instantiated properties or not.

${ }^{19}$ Remember that it is a flexible and cumulative hierarchy. Properties may have a variable arity, which is why in this level 3 state of affairs Diversity 3 was instantiated in four different entities, while on the level 3 fact it was instantiated in three (but could have been instantiated in two, four, five, etc.). A property like Diversity may also combine with different entities, which is why it may be combined with objects, properties, facts and/or states of affairs.

${ }^{20}$ Another apparent loss is the fact that we hoped that our distinction could also be used in an ontology as the one proposed by Armstrong. However, Armstrong argues that his sates of affairs are concrete entities: "Speaking now of my own states of affairs, I'd say that they are concrete (...)" (Armstrong 2009, p.40). This could be said for our first approach to facts, but it does not seem to hold for the second approach which allows higher-level facts (though Armstrong does consider that higher-level states of affairs could be accepted, which could be something worth reviewing in the future).

${ }^{21}$ We have talked about some of its characteristics in the rest of the article. However, in this section we reintroduce some of the notions to explain how the types of the entities in the hierarchy are construed.

22 The hierarchy is controversial. A working mathematician would not take it into account. However, we are developing an ontology which involves logical entities, and the hierarchy gives us a nice way of effectively doing that.

${ }^{23}$ The subscripts to $\kappa$ were put to avoid confusions in the arity of the different level properties.

${ }^{24}$ Even though in this article we maintain that level 0 is the level of concrete objects, in future research we will defend that events also pertain to level 0 . However, since this will most certainly involve changing types, we do not take them into account.

${ }^{25}$ We use $T$ for facts because the properties may be instantiated in any kind of entity, as long as it involves at least one object or fact. We use $\tau$ for states of affairs because they may only be instantiated in properties and/or states of affairs. Thus, $\tau$ presents more restrictions than $T$.

26 This example could be simplified as follows: assume that we are working with the specific state of affairs $\langle$ Existence3, Existence 2$\rangle$ that has as an argument Existence 2 which takes as an argument a property like being mortal; the type, then, is fixed and is: $\langle\langle\langle 3,1\rangle,\langle\langle 2,1\rangle,\langle\langle 1,1\rangle$, $0\rangle\rangle\rangle,\langle\langle 2,1\rangle,\langle\langle 1,1\rangle, 0\rangle\rangle\rangle$. 
${ }^{27}$ We could simplify the types of the properties and say that the type is just $\langle\lambda, \kappa\rangle$. If we did this, the types would be simpler. The facts would be the only entities that have 0 in it, and the states of affairs would not have any 0 in it. However, if we want to defend that - from level 2 and up - there is a different Existence 2 property according to the type of the argument it takes, the alternative we provided is the one to follow. We, thus, maintain the type for all the properties, because we want to type properties in the same way.

28 Obviously, in this framework the terms should be revised, but not the ontological differences we presented.

Principia 20(3): 361-376 (2016). 